\title{
Hyperoxaluria after modern bariatric surgery: case series and literature review
}

\author{
Jared M. Whitson · G. Bennett Stackhouse • \\ Marshall L. Stoller
}

Received: 30 April 2009/Accepted: 6 June 2009/Published online: 2 July 2009

(C) The Author(s) 2009. This article is published with open access at Springerlink.com

\begin{abstract}
Introduction Two recent studies have shown that modern bariatric surgery leads to significant hyperoxaluria and risk of nephrolithiasis. However, neither report evaluates the use or effects of stone risk modifying agents in these patients. We sought to determine the impact of medical management on stone risk profile in patients who have undergone Roux-en-y gastric bypass.

Materials and methods Twenty-four-hour urine collections of all patients referred to a tertiary clinic for nephrolithiasis in the past 4 years were reviewed. Those patients with severe ( $>75 \mathrm{mg} /$ day) hyperoxaluria were identified. Retrospective chart review was performed to identify those patients with a history of bariatric surgery. Student's $t$-test was used to compare mean urinary parameters between bariatric and non-bariatric patients.

Results Out of all stone formers within our 24-h urine collection database, 39 patients had severe hyperoxaluria (oxalate $>75 \mathrm{mg} /$ day). Twenty-six patients had complete information for review. Five patients had a history of bariatric surgery. Compared with non-bariatric patients, those with a history of
\end{abstract}

J. M. Whitson $(\bowtie) \cdot$ G. B. Stackhouse · M. L. Stoller Department of Urology, University of California San Francisco, 400 Parnassus Ave, A-631, San Francisco, CA 94143, USA

e-mail: jwhitson@urology.ucsf.edu bariatric surgery had increased use of oral calcium (80 vs. $28 \%)$ and citrate supplementation (100 vs. $47 \%)$, higher urinary oxalate (129 vs. $91 \mathrm{mg} /$ day) and volume ( 2.9 vs. $2.4 \mathrm{~L} /$ day), lower urinary citrate (390 vs. $800 \mathrm{mg} /$ day) and calcium ( 155 vs. $235 \mathrm{mg} /$ day), and a decreased supersaturation of calcium oxalate (6.7 vs. 11$)$.

Conclusions Appropriate medical management, in particular oral calcium and citrate supplementation, and perhaps most importantly aggressive fluid intake can mitigate some of the effects of enteric hyperoxaluria caused by fat malabsorption after modern bariatric surgery.

Keywords Obesity - Bariatric surgery · Urinary calculi $\cdot$ Calcium oxalate

\section{Introduction}

Obesity is an increasingly prevalent problem in the United States [1]. Seventeen percent of children and $32 \%$ of adults in the US meet the clinical definition of obesity. In adult men and in adolescents of both sexes, these numbers have been climbing over the past 6 years, and in women the rate of obesity seems to be leveling off. The obesity epidemic has been related to sedentary lifestyle, increased use of processed or high caloric foods, and other trends. A prospective study of three large cohorts found that compared to matched 
controls, obese patients were 1.44 (men) and 1.92 (women) times as likely to form stones [2]. Other studies have shown that obese patients have an increased excretion of stone-promoting substances [3].

The newest trend in treatment of morbid obesity is Roux-en-y gastric bypass or gastric banding. The rate at which these procedures are being performed has increased 600\% from 1996 to 2001 [4]. In the 1970s, jejunoileal bypass led to significant complications, including nephrolithiasis, ranging from $11 \%$ at 1 to 5 years [5] to as high as $39 \%$ after 15 years [6]. In contrast, modern bariatric surgery procedures, which are touted to cause less malabsorption than jejunoileal bypass, have been postulated to have less impact on urinary stone risk.

Yet kidney stones were recently found to be one of the top 10 postoperative diagnoses in modern bariatric patients [7]. A separate retrospective study found de novo stone formation in $3.2 \%$ of patients; however, for patients with a history of nephrolithiasis, $31.4 \%$ were found to have recurrent stones after bypass surgery [8]. In addition, hyperoxaluria was recently confirmed to be the most significant abnormality in urinary stone risk profile after modern bariatric surgery [9]. Furthermore, hyperoxaluria has been shown to increase as time from surgery increases [10]. In both of these studies, the patients had low urinary volumes, and in neither study did the authors comment on the use of oral medications to modify stone risk.

Given what has been described as a pending epidemic in nephrolithiasis in patients undergoing modern bariatric surgery, [11] we sought to evaluate the effects of medical management on urinary biochemical data between hyperoxaluric patients with and without a history of bariatric surgery.

\section{Materials and methods}

A retrospective study of urine chemistries was performed. Patients selected for the study had been referred to a urinary stone practice at a tertiary medical center for at least one episode of urolithiasis; many patients had previously undergone 24-h urine collection and/or been placed on some form of medical therapy by the referring urologist. Included patients had submitted at least two outpatient 24-h urine collections (within $\sim 1$ week's time) and a questionnaire to a commercial laboratory (Litholink, Chicago, Ill). As in many practices, we recommend to all of our stone patients to decrease sodium, limit animal protein, and drink sufficient fluids so as to maintain a urine volume around $2 \mathrm{~L} /$ day. We also use the 24-h urine collection to make specific recommendations for dietary change and medical therapy. However, given the known tendency to chronic acidemia, hypocitraturia, and enteric hyperoxaluria in patients who have undergone bariatric surgery, we place all of these patients on 1,200 $\mathrm{mg}$ oral calcium divided up with meals as well as 20 meq potassium citrate three times daily.

Those patients with severe ( $>75 \mathrm{mg} /$ day) hyperoxaluria were identified. Retrospective chart review was performed to determine their medical and surgical histories, especially regarding intestinal surgery and use of medications. An average value (over the two samples submitted by each patient) was determined for each urinary stone risk factor. Urinary $\mathrm{pH}$ was measured using a $\mathrm{pH}$ electrode. Calcium, magnesium, uric acid, creatinine, phosphorous, sodium, potassium, and ammonium were measured using a Beckman autoanalyzer (Beckman Instruments, Brea, CA). Urine supersaturations of calcium oxalate, calcium phosphate, and uric acid were calculated by the iterative computer program, Equil II [12]. In addition, the patient's self-reported information on 19 clinical parameters was included-including family history, personal stone history, and use of stone risk modifying medications. Comparison between groups was made using the Student's $t$-test utilizing commercially available statistical analysis software (SPSS, Chicago, IL).

\section{Results}

We identified 39 patients who had severe hyperoxaluria (oxalate $>75 \mathrm{mg}$ /day), 26 of whom had clinic charts available for review of medical and surgical histories. Five of these 26 patients were found to have a history of Roux-en-y gastric bypass surgery. No bariatric surgery patient reported a history of nephrolithiasis prior to weight reduction surgery. In addition, none of the operations had taken place within the year prior to 24-h urine collection. A single non-bariatric patient had documented Crohn's disease and was 
Table 1 Characteristics of hyperoxaluric patients

\begin{tabular}{llc}
\hline & Non-bariatric $(n=21)$ & Bariatric $(n=5)$ \\
\hline Mean age (years) & 54 & 54 \\
Males (\%) & 76 & 20 \\
Weight (kg) & 78 & 97 \\
Hctz (\%) & 19 & 20 \\
Calcium supp. (\%) & 29 & 100 \\
Citrate supp. (\%) & 48 & 60 \\
Allopurinol (\%) & 10 & 0 \\
\hline
\end{tabular}

excluded. All included non-bariatric patients had presumed dietary or idiopathic hyperoxaluria.

The mean age, weight, and medication use are shown in Table 1. Patient age ranged from 42 to 73 years. Seventy-six percent of the non-bariatric patients were men, although $80 \%$ of the bariatric patients were female. Bariatric patients were heavier than non-bariatric patients (97 vs. $78 \mathrm{~kg}$ ). Nonbariatric and bariatric patients had a similar number of lifetime stone episodes (4.1 vs. 3.6). Oral calcium supplement (80 vs. $28 \%$ ) and potassium citrate (100 vs. $47 \%$ ) use were more common in the bariatric than non-bariatric patients.

Tables 2, 3, and 4 provide a summary of the urinary biochemical data. Those patients with a history of bariatric surgery had a significantly higher chloride

Table 2 Mean 24-h urine parameters by history of bariatric surgery

\begin{tabular}{llll}
\hline & Non-bariatric & Bariatric & $P$-value \\
\hline Ammonium & 41 & 58 & 0.17 \\
Calcium & 240 & 160 & 0.25 \\
Chloride & 190 & 350 & 0.01 \\
Citrate & 800 & 390 & 0.04 \\
Creatinine & 1,900 & 1,600 & 0.36 \\
Magnesium & 130 & 130 & 0.88 \\
Oxalate & 91 & 130 & 0.01 \\
pH & 6.3 & 6.1 & 0.58 \\
Phosphorous & 1.2 & 1.2 & 0.75 \\
Potassium & 93 & 100 & 0.71 \\
Sodium & 210 & 350 & 0.01 \\
Sulfate & 51 & 58 & 0.60 \\
Urea nitrogen & 14 & 13 & 0.77 \\
Uric acid & 0.89 & 0.76 & 0.34 \\
Volume & 2.5 & 2.9 & 0.39 \\
\hline
\end{tabular}

Table 3 Mean 24-h urine concentrations by history of bariatric surgery

\begin{tabular}{llll}
\hline & Non-bariatric & Bariatric & $P$-value \\
\hline Ammonium & 19 & 21 & 0.69 \\
Calcium & 99 & 54 & 0.13 \\
Chloride & 85 & 120 & 0.04 \\
Citrate & 390 & 130 & 0.05 \\
Creatinine & 860 & 590 & 0.11 \\
Magnesium & 57 & 49 & 0.48 \\
Oxalate & 41 & 47 & 0.48 \\
Phosphorous & 0.54 & 0.42 & 0.38 \\
Potassium & 42 & 34 & 0.48 \\
Sodium & 91 & 120 & 0.08 \\
Sulfate & 23 & 19 & 0.57 \\
Urea nitrogen & 6.3 & 4.8 & 0.32 \\
Uric acid & 0.41 & 0.27 & 0.10 \\
\hline
\end{tabular}

(350 vs. $190 P<0.01$ ), a lower citrate (390 vs. 800 $P=0.04)$, higher oxalate $(130$ vs. $91 P<0.01)$, and higher sodium ( 350 vs. $210 P<0.01)$. Urinary volume was $500 \mathrm{~mL}$ greater in the bariatric than non-bariatric group (2.9 vs. $2.4 \mathrm{~L} P=0.36)$.

The concentration of each urinary solute also was calculated and compared between the two groups. The supersaturation of different stone constituents is determined more by solute concentration, rather than net amount excreted per day. These results can be found in Table 3 . Only chloride (120 vs. $85 P=0.04$ ) and citrate (130 vs. $390 P=0.05)$ showed a statistically significant difference. Calcium concentration was almost $50 \%$ less in bariatric patients versus nonbariatric patients, although this did not reach statistical significance (54 vs. $99 P=0.13$ ).

No difference was seen in the supersaturation of uric acid or calcium phosphate. The supersaturation of calcium oxalate was $40 \%$ less in the bariatric patients versus the non-bariatric patients with hyperoxaluria (6.7 vs. $11 P=0.13$ ). These results can be found in Table 4.

Table 4 Mean 24-h urine supersaturations by history of bariatric surgery

\begin{tabular}{llll}
\hline & Non-bariatric & Bariatric & $P$-value \\
\hline SS UA & 0.82 & 0.67 & 0.76 \\
SS CaOx & 11 & 6.7 & 0.13 \\
SS CaPhos & 1.4 & 0.64 & 0.25 \\
\hline
\end{tabular}




\section{Discussion}

Oxalate excretion has been identified as a major risk factor for stone formation for decades. Hyperoxaluria following bariatric surgery was definitively shown to be from increased intestinal oxalate absorption in the early 1980s [13]. In this study, six patients were placed on an oxalate diet, a low-oxalate diet, and total parenteral nutrition. Urinary oxalate on a low-oxalate diet decreased by half, while on total parenteral nutrition, a fourfold decrease in urinary oxalate was found. A complementary study revealed the importance of high oral calcium intake in binding luminal oxalate. The average urinary oxalate doubled when dietary calcium was reduced from 3,000 to $250 \mathrm{mg} /$ day [14]. These studies clearly show that dietary or medical intervention can mitigate some of the effects of bariatric surgery. In an attempt to further define the effects of aggressive medical management on urinary stone risk profile in patients undergoing Roux-en-y gastric bypass, we retrospectively identified and compared hyperoxalurics with and without a history of bariatric surgery.

The most notable finding of this study was that following bariatric surgery, patients had a surprisingly low supersaturation of calcium oxalate of 6.7. This was mainly due to a significantly lower urinary calcium concentration. In fact, the supersaturation of calcium oxalate in our bariatric patients is similar to that found in a previous large cohort study of normal, overweight, and obese stone formers. The obese stone formers had a supersaturation of calcium oxalate (by the EQUIL equation) of 6.14 (men) and 5.97 (women) [15]. Therefore, by comparison, our obese patients after bariatric surgery had a supersaturation of calcium oxalate, which was only $10 \%$ higher. This finding is in contradiction to a study in the early 1980s that looked at 24-h urine collections in obese patients before and both 2 and 5 years after jejunoileal bypass surgery. Following surgery, urinary calcium decreased from baseline, and oxalate excretion was threefold higher, despite calcium supplementation. However, in this study, the relative SS product (by Robertson's equation) increased by a full $40 \%$ following surgery [16].

However, the low supersaturation of calcium oxalate in our bariatric patients with hyperoxaluria is even more surprising given recent data published by Asplin [9]. This found a SS $\mathrm{CaOx}$ in gastric bypass and jejunoileal bypass patients that was nearly twice as high as our value of 6.7. Although our patients had higher urinary oxalate (130 vs. $100 \mathrm{mg} /$ day $)$, higher urinary calcium (160 vs. $140 \mathrm{mg} /$ day), and lower citrate (390 vs. $\sim 400 \mathrm{mg} /$ day), our patients' urinary volume was significantly greater $(2.9$ vs. $1.8 \mathrm{~L})$. This may be the key difference between our bariatric stone formers and those of Asplin. Thus, many of the recommendations that are applicable to the stoneforming public in general, i.e., increased fluid intake, can have a significant impact on stone-forming risk even in this highly selected population.

Similarly, citrate has been studied extensively in weight reduction surgery patients. Citrate is a known inhibitor of the formation of calcium oxalate stones. Bariatric surgery can have profound effects on urinary citrate levels due to chronic metabolic acidosis. A study in jejunoileal bypass patients found a mean urinary citrate of $22 \mathrm{mg} /$ day. These patients subsequently underwent bypass reversal with an increase in urinary citrate to $230 \mathrm{mg} / \mathrm{day}$. Citrate supplementation was initiated, and urinary levels rose further to $550 \mathrm{mg} /$ day [17]. In contrast, we did not see a significant degree of hypocitraturia in our studied bypass patients. This was likely influenced by the fact that all of the bariatric surgery patients were taking potassium citrate at the time of 24-h urine collection. However, urinary citrate levels were still significantly lower in the bariatric patients than non-bariatric patients, despite their higher rate of citrate supplementation. Additionally, compliance is supported by the mean urinary potassium level $(100 \mathrm{mg} /$ day $)$ since for every one $\mathrm{mEq}$ of potassium intake; approximately one $\mathrm{mEq}$ of potassium appears in the urine.

The influence of inhibitors of urinary stone formation also has been studied in weight reduction surgery patients. High oxalate levels in the intestine bind not only calcium, but also magnesium (a stone inhibitor). High calcium oxalate stone indices following jejunoileal bypass have been attributed to both to high urinary oxalate and low urinary magnesium [18], and $80 \%$ of our bariatric patients were on oral calcium, which likely lowered both oxalate absorption and oxalate available for magnesium binding. Therefore, it is not surprising that our study found no difference in magnesium levels between hyperoxaluric patients with or without a history of bariatric surgery.

Our study included a large number of patients with hyperoxaluria, but was limited by its retrospective nature and small size of specific patients with a 
history of bariatric surgery. Since most patients were seen after evaluation and treatment by an outside urologist, we were not able to examine an individual patient's response to any given therapy, i.e., a change in 24-h urine over time. Furthermore, although we were able to determine from chart review that all of the bypass procedures had taken place over a year prior to 24-h urine collection, the exact dates of surgery could not be determined. Additionally, the comparison groups differed in distribution of gender, as more of the bariatric patients were women. Men in general have higher urine SS CaOx, which could have brought the results closer to the null hypothesis. While it is reassuring that none of our bariatric patients had renal insufficiency to suggest oxalate nephropathy, a recent study reported on two patients (8.7\% of the study cohort) with renal failure presumed to be due to hyperoxaluria after Roux-eny gastric bypass [19].

Future studies could focus on 24-h urine collections in patients before and after bariatric surgery. This kind of internal control could factor in each patient's inherent stone-forming risk, with the additional risks of obesity and subsequent bariatric surgery and weight loss. This could be performed in a prospective fashion in coordination with a bariatric surgery center. These patients undergo extensive evaluations with sometimes multiple follow-up visits prior to surgery, and the opportunity likely exists for enrollment in a clinical trial. Because of the dramatic increase in the numbers of these procedures, as well as the potential difficulties in performing stone operations in these patients, we need to know significantly more about the exact risk and nature of stone formation in these patients.

\section{Conclusions}

Despite the anticipated significantly higher oxalate levels, compared with other hyperoxaluric patients, modern bariatric surgery patients had a dramatically lower supersaturation of calcium oxalate, predominantly due to higher urinary volume and lower urinary calcium excretion. Appropriate medical management, in particular oral calcium supplementation and aggressive fluid intake may mitigate some of the effects of enteric hyperoxaluria after modern bariatric surgery.
Open Access This article is distributed under the terms of the Creative Commons Attribution Noncommercial License which permits any noncommercial use, distribution, and reproduction in any medium, provided the original author(s) and source are credited.

\section{References}

1. Ogden CL, Carroll MD, Curtin LR et al (2006) Prevalence of overweight and obesity in the United States, 1999-2004. JAMA 295:1549-1555. doi:10.1001/jama.295.13.1549

2. Taylor EN, Stampfer MJ, Curhan GC (2005) Obesity, weight gain, and the risk of kidney stones. JAMA 293:455462. doi:10.1001/jama.293.4.455

3. Powell CR, Stoller ML, Schwartz BF et al (2000) Impact of body weight on urinary electrolytes in urinary stone formers. Urology 55:825-830. doi:10.1016/S0090-4295(99) 00617-2

4. Livingston EH (2004) Procedure incidence and in-hospital complication rates of bariatric surgery in the United States. Am J Surg 188:105-110. doi:10.1016/j.amjsurg.2004.03. 001

5. Clayman RV, Williams RD (1979) Oxalate urolithiasis following jejunoileal bypass. Surg Clin North Am 59: 1071-1077

6. Annuk M, Backman U, Holmgren K et al (1998) Urinary calculi and jejunoileal bypass operation. A long-term follow-up. Scand J Urol Nephrol 32:177-180. doi:10.1080/ 003655998750015539

7. Encinosa WE, Bernard DM, Chen CC et al (2006) Healthcare utilization and outcomes after bariatric surgery. Med Care 44:706-712. doi:10.1097/01.mlr.0000220833. 89050.ed

8. Durrani O, Morrisroe S, Jackman S et al (2006) Analysis of stone disease in morbidly obese patients undergoing gastric bypass surgery. J Endourol 20:749-752. doi:10.1089/end. 2006.20.749

9. Asplin JR, Coe FL (2007) Hyperoxaluria in kidney stone formers treated with modern bariatric surgery. J Urol 177: 565-569. doi:10.1016/j.juro.2006.09.033

10. Sinha MK, Collazo-Clavell ML, Rule A et al (2007) Hyperoxaluric nephrolithiasis is a complication of roux-en-Y gastric bypass surgery. Kidney Int 72:100-107. doi:10.1038/ sj.ki.5002194

11. Miller NL (2008) Modern bariatric surgery and nephrolithiasis-are we on the verge of a new epidemic? J Urol 179:403-404. doi:10.1016/j.juro.2007.11.006

12. Werness PG, Brown CM, Smith LH et al (1985) EQUIL2: a BASIC computer program for the calculation of urinary saturation. J Urol 134:1242-1244

13. Nordenvall B, Backman L, Larsson L (1981) Oxalate metabolism after intestinal bypass operations. Scand J Gastroenterol 16:395-399

14. Stauffer JQ (1977) Hyperoxaluria and calcium oxalate nephrolithiasis after jejunoileal bypass. Am J Clin Nutr 30:64-71

15. Siener R, Glatz S, Nicolay C et al (2004) The role of overweight and obesity in calcium oxalate stone formation. Obes Res 12:106-113. doi:10.1038/oby.2004.14 
16. Scholz D, Schwille PO, Husemann B et al (1982) Mineral metabolism during prolonged oral calcium substitution after jejuno-ileal bypass for morbid obesity. Klin Wochenschr 60:803-809. doi:10.1007/BF01721145

17. Dhar NB, Grundfest S, Jones JS et al (2005) Jejunoileal bypass reversal: effect on renal function, metabolic parameters and stone formation. J Urol 174:1844-1846. doi: 10.1097/01.ju.0000177079.56949.1a (discussion 6)
18. Holm CN, Hessov I (1981) Effect of calcium treatment on urinary stone index after intestinal bypass for obesity. Digestion 22:255-258. doi:10.1159/000198665

19. Nelson WK, Houghton SG, Milliner DS et al (2005) Enteric hyperoxaluria, nephrolithiasis, and oxalate nephropathy: potentially serious and unappreciated complications of roux-en-Y gastric bypass. Surg Obes Relat Dis 1:481-485. doi:10.1016/j.soard.2005.07.002 